\title{
Review
}

\section{Non-Pharmacological Interventions in Refractory Angina}

\author{
Noman Ali, PhD"*; Peysh Patel, MA \\ "Denotes joint authorship \\ Department of Cardiology, Leeds General Infirmary, Leeds, LSI 3EX, UK
}

*Corresponding author

Noman Ali, PhD

Department of Cardiology, Leeds General Infirmary, Leeds, LSI 3EX, UK; E-mail: Nomanali456@doctors.org.uk

\section{Article information}

Received: March 3 ${ }^{\text {rd }}, 2018$; Revised: April 6 ${ }^{\text {th }}$ 2018; Accepted: April 10 ${ }^{\text {th }}$, 20I8; Published: April II ${ }^{\text {th }}$ 20I8

\section{Cite this article}

Ali N, Patel P. Non-pharmacological interventions in refractory angina. Heart Res Open J. 20I8; 5(I): I-7. doi: I0.17I40/HROJ-5-I46

\section{ABSTRACT}

Refractory angina (RA) is defined by intractable cardiac chest pain despite standard medical therapy and revascularisation. Symptoms are associated with significant morbidity and compromised quality of life (QoL). Various methods of interventional pain management can be considered for the complex care of these cohorts. This review explores postulated mechanisms and evidence base underpinning therapeutic strategies such as cognitive behavioural therapy (CBT), cardiac rehabilitation, stellate ganglion blockade (SGB), enhanced external counterpulsation (EECP) and coronary sinus reducer (CSR) implantation. Use of these modalities is dependent on the local provision, but suitability is best assessed by specialist RA services using an individualised, multidisciplinary framework.

\section{Keywords}

Refractory angina (RA); Enhanced external counterpulsation (EECP); Cardiac rehabilitation.

\section{Abbreviations}

RA: Refractory Angina; EECP: Enhanced External Counterpulsation; CBT: Cognitive Behavioural Therapy; CSR: Coronary Sinus Reducer; SGB: Stellate Ganglion Blockade; OMT: Optimal Medical Therapy; CAD: Coronary Artery Disease; QoL: Quality of Life; ESC: European Society of Cardiology; GTN: Glyceryl Tri-nitrate; HCN: Hyperpolarisation-activated Cyclic Nucleotide-gated; PCI: Percutaneous Coronary Intervention; CABG: Coronary Artery Bypass Grafting; RCTs: Randomised-Controlled Trials; CPRS: Complex Regional Pain Syndrome; LA: Local Anaesthetic; IABP: Intra-Aortic Balloon Pump; VEGF: Vascular Endothelial Growth Factor; TNF- $\alpha$ : Tumour Necrosis Factor- $\alpha$; VCAM-1: Vascular Cell Adhesion Molecule-1; SCS: Spinal Cord Stimulation; TENS: Transcutaneous Electrical Neural Stimulation; ESMR: Extra Corporeal Shockwave Myocardial Revasculariusation; RIPC: Remote Ischemic Preconditioning.

\section{INTRODUCTION}

$\mathrm{R}$ efractory angina (RA) is a chronic condition characterised by persistent anginal symptoms despite optimal medical therapy (OMT) and revascularisation. ${ }^{1}$ In order to fulfil the conventional definition of this disorder, arterial insufficiency in the setting of coronary artery disease (CAD) needs to be demonstrated, and reversible myocardial ischaemia should be clinically established as the cause of the symptoms. ${ }^{1}$

Although, definitive data pertaining to the prevalence of $\mathrm{RA}$ is scarce, there is a general recognition that it is a growing problem, and one that results in significant public health burden. ${ }^{2}$ The debilitating nature of the symptoms associated with RA can result in a marked detriment to quality of life (QoL), with sufferers often requiring recurrent hospital admissions. Moreover, patients may develop a maladaptive psychological response to their disorder, with heightened and dysregulated perception of their symptoms. This often results in increased utilization of services in the secondary care setting. ${ }^{3}$ In 2013, a prospective analysis of 1200 patients was published which demonstrated the long-term mortality associated with RA to be lower than previously reported. ${ }^{4}$ As such, in recent years the focus of treatment has shifted towards alleviation of symptoms and improved QoL. However, achieving these aims can be challenging due to the complex interplay between physical and psychological factors in RA. This review summarises the 
therapeutic options available for the treatment of RA, with a focus on the non-pharmacological interventions that are used by specialists.

\section{MEDICAL THERAPY AND REVASCULARISATION}

Pharmacotherapy and revascularisation remain the cornerstone initial treatments for patients with RA, as they do for patients with stable angina. The current European Society of Cardiology (ESC) guidelines for management of stable CAD recommend that all patients should be treated with low-dose aspirin and a statin for the purpose of event prevention. ${ }^{5}$ Vasodilating drugs such as sublingual glyceryl trinitrate (GTN), and rate-limiting agents such as beta-blockers or non-dihydropyridine calcium channel blockers, should also be prescribed as first line antianginal agents. If symptoms persist despite these measures, additional vasodilators such as long-acting nitrates or nicorandil can be used. ${ }^{5}$ More novel agents are also available, including ivabradine and ranolazine. Ivabradine specifically inhibits hyperpolarisation-activated cyclic nucleotidegated $(\mathrm{HCN})$ channels, thereby decreasing the 'funny' pacemaker current (If) within the sinoatrial node. ${ }^{6}$ The effect is an antianginal action via reduction in HR, but with no effect on inotropy, vascular resistance or blood pressure. Meanwhile, ranolazine achieves its effects on myocytes via inhibition of the late phase of the inward sodium current (INa) during Phase 0 of action potentials. ${ }^{7}$ This is thought to reduce ventricular stiffness during diastole and hence improve coronary perfusion. ${ }^{8}$

Revascularisation, either via percutaneous coronary intervention (PCI) or coronary artery bypass grafting (CABG), may also provide effective symptom control in certain patients. The ESC suggests consideration of revascularisation in patients who have significant $\mathrm{CAD}$, demonstrate $>10 \%$ ischaemic myocardium on functional testing and remain symptomatic despite OMT. ${ }^{5}$ In the context of stable angina, revascularisation is mainly undertaken for symptomatic rather than prognostic reasons. The exception to this is amongst patients who have significant left main stem/proximal left anterior descending artery disease or multi-vessel disease with impaired left ventricular systolic dysfunction, where revascularisation is thought to provide mortality benefit. ${ }^{9}$

\section{COGNITIVE BEHAVIOURAL THERAPY}

Due to the profound psychological effect of RA on patients, cognitive-behavioural therapy (CBT) is often used to help patients manage their symptoms more effectively. It is usually delivered by clinical nurse specialists and clinical psychologists, and the focus of sessions are to help patients develop more effective coping strategies, primarily based on a greater understanding of what angina is, and by dealing with associated misconceptions. Patients are educated on the concept that management isn't solely limited to pharmacotherapy and revascularisation, but also involves a fundamental alteration in thought processes involved with symptom recognition and interpretation. Particular emphasis is placed on lifestyle alterations including setting of realistic goals, relaxation exercises, energy conservation and sleep quality enhancement. Sessions are run in small groups, providing an informal forum to share and engage with experiences of others to achieve a collective goal of more ef- fective self-management of this chronic condition.

Previous studies assessing the benefits of psychotherapy have been encouraging. A meta-analysis of randomised-controlled trials (RCTs) examining self-management programmes for chronic angina, including RA, suggested that intervention resulted in approximately three fewer episodes of angina per week. This was accompanied by a reduction in usage of glyceryltrinitrate (GTN) spray, a vasodilator commonly prescribed as a sublingual preparation for prophylaxis and treatment of anginal symptoms. ${ }^{10}$ Furthermore, our group has also published data demonstrating that CBT has a sustainable impact on improving QoL for patients with RA. ${ }^{11}$ Nonetheless, CBT is not yet widely accessible and usually necessitates formal referral and subsequent review by a specialist RA service.

Novel research has also explored the possibility that RA ought to be conceptualised as a chronic pain disorder, with integration of physical, psychological and educational treatment modalities. ${ }^{12}$ Consistent with this notion, it has been postulated that the maladaptive cognitive responses to pain may be ameliorated through mindfulness meditation. A standardised program has been shown to have a positive impact on pain management and clinical sequelae in patients with chronic pain, but its role in the context of RA is yet to be established. ${ }^{13}$

\section{CARDIAC REHABILITATION}

Rehabilitation strategies reduce psychological symptom burden, particularly anxiety and depression, in the context of ischaemic heart disease. They are also designed to enhance functional capacity and aerobic conditioning. However, its role has been less extensively explored in the context of RA and this may relate to concerns regarding adverse events in patients with inherently high risk. A pilot study has shown that a 2-month outpatient rehabilitation program improved exercise tolerance without significant deterioration (albeit no improvement) in anginal symptoms. ${ }^{14}$ The study was flawed in its design and under-powered. Further validation is therefore required before it can be considered for incorporation into clinical practice, and currently, the concept is not specifically addressed in management guidelines.

\section{STELLATE GANGLION BLOCKADE}

Afferent sympathetic fibres have been implicated in the pathophysiology of various conditions, including complex regional pain syndrome (CPRS). ${ }^{15}$ A neuronal blockade is postulated to interrupt adrenergic hypersensitivity and positive feedback circuits that ordinarily augment central excitability. The notion of treating cardiac chest pain by modulation of sympathetic afferents was first postulated by Frank in 1899. During the next few decades, various approaches were explored that were associated with effective alleviation of symptoms but limited by irreversible sequelae. ${ }^{16}$

It was subsequently hypothesised that application of local anaesthetic (LA) in the region of the middle cervical sympathetic ganglion or the cervicothoracic (stellate) sympathetic ganglion may provide a more effective strategy for autonomic modulation. ${ }^{17}$ This 
concept is now conventionally termed 'stellate ganglion blockade' (SGB). Initial techniques involved a puncture between the carotid artery and the cricoid cartilage (C6 level), which was associated with high rates of complications such as retropharyngeal haematoma and oesophageal rupture. ${ }^{18}$ These procedural risks have been largely circumvented by use of ultrasound guidance to enable appropriate needle tip placement. The type of LA used can vary depending on required duration of effect and indication, with examples including alcohol and bupivacaine. A successful outcome is traditionally defined by the development of transient Horner's syndrome, a classic triad of miosis (constricted pupil), partial ptosis (eyelid drooping) and ipsilateral anhidrosis (hemifacial loss of sweating). It is typically associated with concomitant increase in blood flow, skin temperature and loss of the galvanic skin response.

The first clinical trial on angina sufferers was in 1966, where SGB was shown to be superior to placebo in reducing exertional symptoms. ${ }^{19}$ A more recent single-centre prospective study has shown SGB to be both safe and efficacious in the management of RA, with a mean pain relief duration of 3.5 weeks. ${ }^{20}$ Mild, fully reversible procedural complications occurred only in a small minority $(3 \%)$. However, the long-term benefits of temporary sympathectomy are not well characterised. A case report has described benefits of SGB in a patient with end-stage CAD and RA that lasted months to years, but this may be associated with adverse sequelae including permanent Horner's syndrome. ${ }^{21}$

Overall, SBG can be considered as an alternative interventional strategy in patients who have intractable anginal symptoms. However, the evidence base for this procedure is lacking and further RCTs are warranted to establish whether it should be incorporated into routine clinical practice.

\section{ENHANCED EXTERNAL COUNTERPULSATION (EECP)}

Whilst a number of non-pharmacological therapeutic options have been used in the management of RA, the current ESC guidelines for the management of stable CAD highlighted Enhanced External Counterpulsation (EECP) as the modality with the best evidence base (Table 1). ${ }^{5}$ EECP is a technique that works on the principle of using aortic counterpulsation in order to augment diastolic blood flow. Pressure cuffs are wrapped around the lower limbs of patients and inflated at the onset of diastole. The resulting increase indiastolic blood pressure has the effect of improving coronary perfusion pressure during diastole. The rapid deflation of the cuffs at the onset of systole also serves to reduce vascular resistance, causing a reduction in cardiac workload. ${ }^{22}$ The combined effect is analogous to that of an intra-aortic balloon pump (IABP), albeit in a non-invasive fashion.

Clinical trials utilising EECP in patients with RA have been encouraging. The MUST-EECP study was an RCT that showed EECP to be associated with a significant decrease in angina frequency amongst patients with RA, as well as an improvement in time to ST-segment depression on stress testing. ${ }^{23}$ Subsequently, a number of studies have corroborated these findings, with meta-analyses demonstrating that use of EECP is associated with improvements in anginal symptom burden ${ }^{24,25}$ and myocardial perfusion. ${ }^{26}$ In addition to the multitude of studies demonstrating the symptomatic benefit of EECP, the technique is also of proven effectiveness in reducing hospital expenditure. ${ }^{27}$ Data collected from phase II of the International EECP Patient Registry (IEPRII) demonstrated a significant reduction in the rate of hospitalisation following EECP, resulting in an annual cost saving of $\$ 17,074$ per patient. Based upon this body of evidence, EECP has become established as a treatment for RA in North America, China, Russia, India and the Middle East.

Whilst a significant body of evidence exists in support of EECP, there remains uncertainty regarding the underpinning mechanisms. A number of theories have been postulated, which predominantly pertain to two closely intertwined concepts; improved endothelial function and augmented angiogenesis. ${ }^{22}$ The

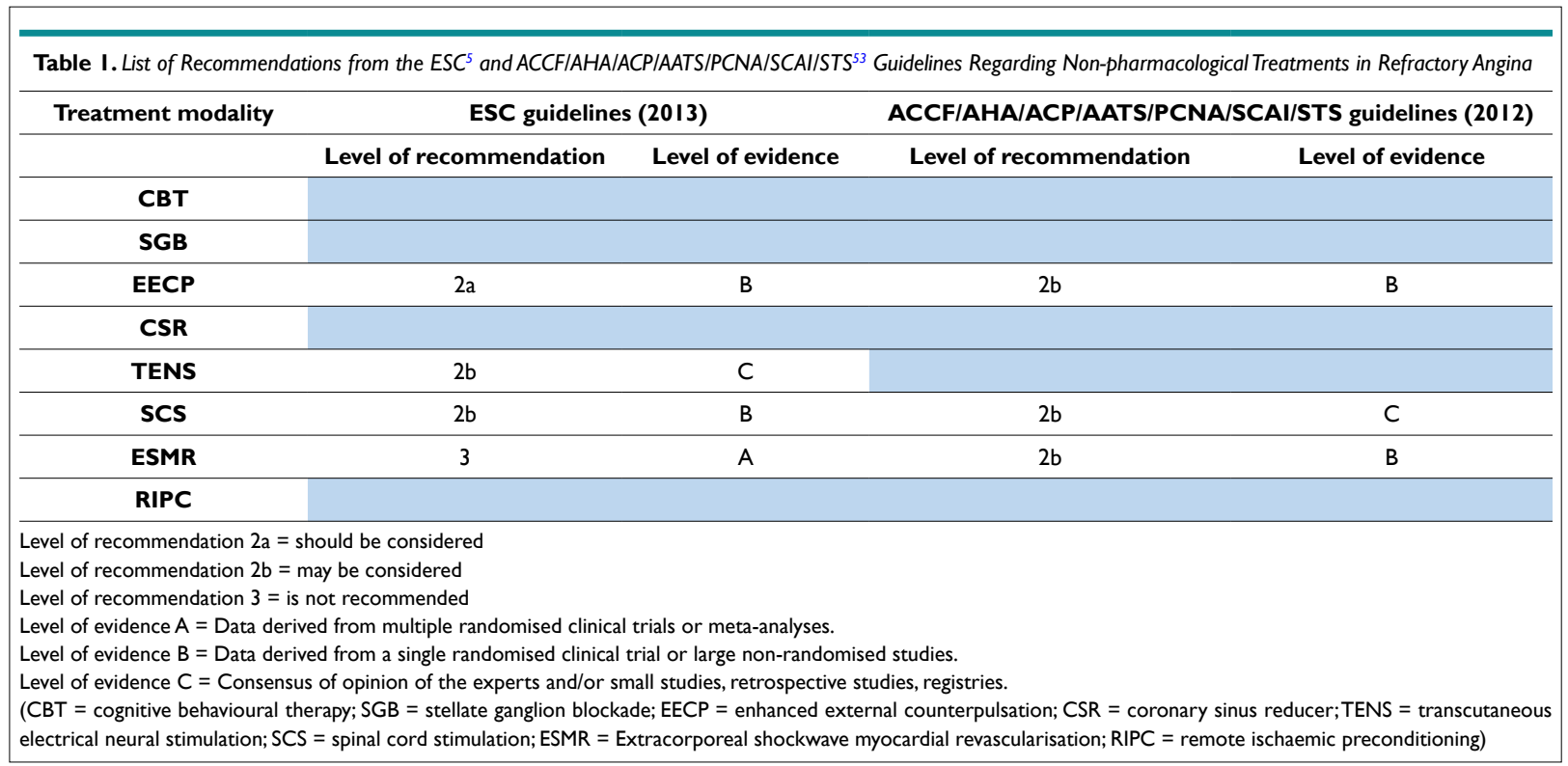


raised diastolic pressure resultant from EECP has been demonstrated to be associated with increased shear stress, which can stimulate pro-angiogenic factors such as the potent vasodilator nitric oxide (NO) and vascular endothelial growth factor (VEGF). ${ }^{28,29}$ The subsequent increase in angiogenesis is hypothesised to be responsible for increased collateral formation within the coronary circulation. EECP has also been shown to improve indices of endothelial dysfunction, with studies demonstrating EECP to be associated with significant improvements in reactive hyperaemiaperipheral arterial tonometry ${ }^{30}$ and flow-mediated vasodilatation. ${ }^{31}$ Furthermore, following EECP, patients with CAD have been noted to have a significant increase in plasma NO concentrations and a concomitant decrease in plasma concentrations of the vasoconstrictor endothelin-1. ${ }^{32}$ Finally, EECP is associated with reductions in pro-inflammatory cytokines such as tumour necrosis factor- $\alpha$ (TNF- $\alpha$ ) and vascular cell adhesion molecule-1 (VCAM-1), which are known to have deleterious effects on endothelial function. Taken together, these mechanisms provide a potential explanation for observed improvements in perfusion scan ischaemia associated with EECP, ${ }^{33}$ and thus may also explain the consistent symptomatic improvements seen amongst patients in clinical trials as well as real-world cohorts.

\section{CORONARY SINUS REDUCER (CSR) IMPLANTATION}

A promising, relatively recent development in the field of RA is the use of coronary sinus reducer (CSR) implantation for patients who have no further revascularisation options but demonstrate inducible ischaemia on functional testing. A CSR in an hour-glass shaped, balloon-expandable, stainless-steel device which is percutaneously implanted into the coronary sinus. It produces a local narrowing of venous drainage which results in increased upstream pressure within the sinus. This is postulated to redistribute collateral flow from the non-ischaemic myocardium to underperfused, ischaemic regions. COSIRA, a multi-centre, prospective, randomised, double-blinded trial, was a landmark study which assessed the safety and efficacy of the CSR. ${ }^{34}$ Patients were included if they had RA and reversible ischaemia in the anterior or lateral wall of the left ventricle. In comparison with a sham procedure, CSR implantation was associated with improved anginal symptom burden and QoL at 6-month follow-up.

Whilst the use of CSR as a treatment for RA is relatively novel, the underlying concept is not; improvement in anginal symptoms and functional status associated with partial occlusion of the coronary sinus was described as far back as 1954 by Beck and Leighninger following surgical ligation. ${ }^{35-39}$ The physiological basis for improvement in anginal symptoms via narrowing of the coronary sinus remains incompletely understood. It is thought that patients with CAD develop dysfunction of a sympathetically-mediated vasoconstrictive response in sub-epicardial vessels, which would otherwise serve to increase blood flow to sub-endocardial capillaries during periods of heightened myocardial oxygen demand. ${ }^{40}$ It is postulated that the resulting sub-endocardial ischaemia is contributory to the pathogenesis of RA. ${ }^{41}$ Canine studies have demonstrated that increasing coronary venous pressure by sinus occlusion assists in redistributing blood from the less ischaemic epicardium to endocardium, thus reducing myocardial isch- aemia. ${ }^{42}$ Subsequently, clinical trials in human subjects have shown a significant reduction in inducible ischaemia following CSR implantation. ${ }^{43,44}$ The technique is now being carried out in a number of centres worldwide, and results from the ongoing REDUCER-I registry are anticipated to provide further evidence with which to assess its efficacy in a real-world population. ${ }^{45}$

\section{OTHER TREATMENT OPTIONS}

Wall and Melzack's 'gate-control' pain theory, published in 1965, has proven to be one of the most seminal pieces of work in the field of chronic pain management. It proposed that pain signals, which are mediated via small nociceptive fibres, can be inhibited by activation of large, non-nociceptive afferent neurones. ${ }^{46}$ This discovery has led to the development of techniques such as spinal cord stimulation (SCS) and transcutaneous electrical neural stimulation (TENS), both of which have been used in the management of RA. SCS uses electrodes implanted within the spinal cord, whereas TENS uses pads placed on the skin. In both cases, low voltage electric current is used to modulate nociceptive signalling and replace pain with paraesthesia. The ESC recommends that both modalities may be considered for the treatment of RA (Table 1). ${ }^{5}$ There have been encouraging results from trials using SCS, with improvements noted in symptom burden and QoL. ${ }^{48-50}$ There is less evidence supporting TENS as an effective treatment for RA, although case series have described its use to be associated with a reduction in anginal symptoms. ${ }^{51}$

Extra corporeal shockwave myocardial revascularisation (ESMR) has also been used in the treatment of RA. Low-intensity shockwaves are targeted at ischaemic myocardium under echocardiographic guidance, and it is thought that the cavitation effect induces local shear stress, promoting expression of pro-angiogenic factors such as VEGF and NO. The net result is increased capillary density and regional myocardial blood flow. ${ }^{52}$ A number of small trials have demonstrated improved symptoms in patients with RA treated with ESMR, although current ESC guidelines do not advocate its use (Table 1$).^{5}$

Remote ischemic preconditioning (RIPC) is a technique which has been studied in animal models, and is now being investigated in humans. The concept is that an organ or tissue can be protected against infarction by use of repetitive, short episodes of ischemia at a remote site. The discovery was made following the observation that in a canine model, short periods of ischemia and reperfusion in the circumflex coronary artery preconditioned myocardium outside of the occluded vasculature. ${ }^{53}$ RIPC has not been extensively studied in RA, but patients are currently being recruited to an RCT which plans to examine its effect. ${ }^{54}$ Patients randomised to the intervention arm of the trial will receive twice daily episodes of upper limb ischaemia/reperfusion delivered by a blood pressure cuff on each arm for a period of 30 days.

\section{CONCLUSIONS}

RA is a chronic disease associated with debilitating morbidity and compromised QoL. Whilst medical therapy with antianginal agents remain the cornerstone of treatment, it is often insufficient. As 
such, non-pharmacological interventions are required. In the last few decades, a number of modalities have been developed including pragmatic rehabilitation via CBT, modulation of pain pathways via stellate ganglion blockade and anti-ischaemic therapies such as EECP and CSR implantation. It is being increasingly recognised that the best results are achieved via combination therapy with more than one of these modalities. Dedicated specialist RA services employing a multidisciplinary approach have been developed, and preliminary data suggest that such an approach may improve QoL and clinical outcomes for RA patients. ${ }^{55}$

\section{CONFLICT OF INTERESTS}

The authors declare that they have no conflicts of interest.

\section{REFERENCES}

1. Mannheimer C, Camici P, Chester MR, et al. The problem of chronic refractory angina; report from the ESC Joint Study Group on the Treatment of Refractory Angina. Eur Heart J. 2002; 23: 335370. doi: 10.1053/euhj.2001.2706

2. Banai S, Ben Muvhar S, Parikh KH, et al. Coronary sinus reducer stent for the treatment of chronic refractory angina pectoris. $J \mathrm{Am}$ Coll Cardiol. 2007; 49: 1783-1789. doi: 10.1016/j. jacc.2007.01.061

3. Moore RKG, Groves DG, Bridson JD, et al. A brief cognitivebehavioural intervention reduces hospital admissions in refractory angina patients. J Pain Symptom Manage. 2007; 33: 310-316. doi: 10.1016/j.jpainsymman.2006.10.009

4. Henry TD, Satran D, Hodges JS, et al. Long-term survival in patients with refractory angina. Eur Heart J. 2013; 34: 2683-2688. doi: 10.1093/eurheartj/eht165

5. European Society of Cardiology guidelines for the management of stable coronary artery disease: The Task Force on the management of stable coronary artery disease of the European Society of Cardiology (ESC). Eur Heart J. 2013; 34: 2949-3003. doi: 10.1093/ eurheartj/eht296

6. Psotka MA, Teerlink JR. Ivabradine: Role in the chronic heart failure Armamentarium. Circulation. 2016; 133(21): 2066-2075. doi: 10.1161/CIRCULATIONAHA.115.018094

7. Keating GM. Ranolazine: A review of its use as add-on therapy in patients with chronic stable angina pectoris. Drugs. 2013; 73(1): 55-73. doi: $10.1007 / s 40265-012-0005-z$

8. Hasenfussand G, Maier LS. Mechanism of action of the new anti-ischemia drug ranolazine. Clin Res Cardiol. 2008; 97(4): 222226. doi: 10.1007/s00392-007-0612-y

9. Wijns W, Kolh P, Danchin N, et al. Guidelines on myocardial revascularization. Eur Heart J. 2010; 31: 2501-2555. doi: 10.1016/j. ejcts.2010.08.019

10. McGillion M, Arthur H, Victor JC, et al. Effectiveness of psychoeducational interventions for improving symptoms, health-related quality of life, and psychological well-being in patients with stableangina. Curr Cardiol Rev. 2008; 4: 1-11. doi: $10.2174 / 157340308783565393$

11. Patel PA, Khan M, Yau C, et al. The short- and long-term impact of psychotherapy in patients with chronic, refractory angina. Br J Cardiol. 2016; 23: 57-60. doi: 10.5837/bjc.2016.019

12. Pak N, Devcich DA, Johnson MH, et al. Is refractory angina pectoris a form of chronic pain? A comparison of two patient groups receiving spinal cord stimulation therapy. N Z Med J. 2014; 127(1391): 52-61.

13. La Cour P, Petersen M. Effects of mindfulness medication on chronic pain: A randomized controlled trial. Pain Med. 2015; 16(4): 641-652. doi: 10.1111/pme.12605

14. Asbury EA, Webb CM, Probert H, et al. Cardiac rehabilitation to improve physical functioning in refractory angina: A pilot study. Cardiology. 2012; 122: 170-177. doi: 10.1159/000339224

15. Drummond PD, Finch PM, Skipworth S, et al. Pain increases during sympathetic arousal in patients with complex regional pain syndrome. Neurology. 2001; 57(7): 1296-1303. doi: 10.1212/ WNL.57.7.1296

16. Meller ST, Gebhart GF. A critical review of the afferent pathways and the potential chemical mediators involved in cardiac pain. Neuroscience. 1992; 48(3): 501-524. doi: 10.1016/03064522(92)90398-L

17. Gask GE. The surgery of the sympathetic nervous system. $\mathrm{Br}$ J Surg. 1993; 21: 113-130.

18. Stanton-Hicks M. Complications of sympathetic blocks for extremity pain. Tech Reg Anesth Pain Manage. 2007; 11(3): 148-151.

19. Wiener L, Cox JW. Influence of stellate ganglion block on angina pectoralis and the post-exercise electrocardiogram. Am J Med Sci. 1966; 252(3): 289-295.

20. Moore R, Groves D, Hammond C, et al. Temporary sympathectomy in the treatment of chronic refractory angina. J Pain Symptom Manage. 2005; 30(2): 183-191. doi: 10.1016/j.jpainsymman.2005.02.016

21. Chester M, Hammond C, Leach A. Long-term benefits of stellate ganglion block in severe chronic refractory angina. Pain. 2000; 87(1): 103-105.

22. Arora RR, Shah AG. The role of enhanced external counterpulsation in the treatment of angina and heart failure. Can J Cardiol. 2007; 23(10): 779-781.

23. Arora RR, Chou TM, Jain D, et al. The multicenter study of enhanced external counterpulsation (MUST-EECP): Effect of EECP on exercise-induced myocardial ischemia and anginal ep- 
isodes. J Am Coll Cardiol. 1999; 33(7): 1833-1840. doi: 10.1016/ S0735-1097(99)00140-0

24. Zhang C, Liu X, Wang X, et al. Efficacy of enhanced external counterpulsation in patients with chronic refractory angina on canadian cardiovascular society (CCS) angina class. Medicine (Baltimore). 2015; 94(47): 2002. doi: 10.1097/MD.0000000000002002

25. Barsness G, Feldman AM, Holmes DR Jr., et al. The international EECP patient registry (IEPR): Design, methods, baseline characteristics, and acute results. Clin Cardiol. 2001; 24(6): 435-442. doi: $10.1002 /$ clc.4960240604

26. Qin X, Deng Y, Wu D, et al. Does enhanced external counterpulsation (EECP) significantly affect myocardial perfusion?: A systematic review \& meta- analysis. PLoS One. 2016; 11(4): e0151822. doi: 10.1371/journal.pone.0151822

27. Lawson WE, Hui JC, Kennard ED, et al. Enhanced external counterpulsation is cost-effective in reducing hospital costs in refractory angina patients. Clin Cardiol. 2015; 38(6): 344-349. doi: $10.1002 /$ clc. 22395

28. Masuda D, Nohara R, Hirai T, et al. Enhanced external counterpulsation improved myocardial perfusion and coronary flow reserve in patients with chronic stable angina; Evaluation by $13 \mathrm{~N}$ ammonia positron emission tomography. Eur Heart J. 2001; 22: 1451-1458. doi: 10.1053/euhj.2000.2545

29. Masuda D, Nohara K, Kataoka K, et al. Enhanced external counterpulsation promotes angiogenesis factors in patients with chronic stable angina. Circulation. 2001; 104: II445. doi: 10.1053/ euhj.2000.2545

30. Bonetti PO, Barsness GW, Keelan PC, et al. Enhanced external counterpulsation improves endothelial function in patients with symptomatic coronary artery disease. J Am Coll Cardiol. 2003; 41: 1761-1768. doi: 10.1016/S0735-1097(03)00329-2

31. Braith RW, Conti CR, Nichols WW, et al. Enhanced external counterpulsation improves peripheral artery flow mediated dilatation in patients with chronic angina: A randomized sham-controlled study. Circulation. 2010; 122: 1612-1620. doi: 10.1161/CIRCULATIONAHA.109.923482

32. Davies PF. Flow-mediated endothelial mechanotransduction. Physiol Rev. 1995; 75: 519-560.

33. Eslamian F, Aslanabadi N, Mahmoudian B, et al. Therapeutic effects of enhanced external counterpulsation on clinical sumptoms, echocardiographic measurements, perfusion scan parameters and exercise tolerance test in coronary artery disease patients with refractory angina. Int J Med Sci Public Health. 2013; 2: 179-187.

34. Verheye S, Jolicoeur EM, Behan MW, et al. Efficacy of a device to narrow the coronary sinus in refractory angina. $N$ Engl J Med. 2015; 372(6): 519-527.
35. Beck CS, Leighninger DS. Operations for coronary artery disease. J Am Med Assoc. 1954; 156: 1226-1233. doi: 10.1001/ jama.1954.02950130006002

36. Beck CS, Leighninger DS. Scientific basis for the surgical treatment of coronary artery disease. J Am Med Assoc. 1955; 159: 1264 1271. doi: 10.1001/jama.1955.02960300008003

37. Sandler G, Slesser BV, Lawson CW. The Beck operation in the treatment of angina pectoris. Thorax. 1967; 22: 34-37.

38. Wising PJ. The Beck-1 operation for angina pectoris: Medical aspects. Acta Med Scand. 1963; 174: 93-98. doi: 10.1111/j.09546820.1963.tb07895.x

39. Brofman BL. Medical evaluation of the Beck operation for coronary artery disease. I Am Med Assoc. 1956; 162: 1603-1606.doi: 10.1001/jama.1956.02970350019005

40. Crea F, Galassi AR, Kaski JC, et al. Effect of theophylline on exercise-induced myocardial ischemia. Lancet. 1989; 1: 683-686. doi: 10.1016/S0140-6736(89)92204-6

41. Benedetto D, Abawi M, Stella PR, et al. Percutaneous device to narrow the coronary sinus: Shifting paradigm in the treatment of refractory angina? A review of the literature. Front Cardiovasc Med. 2016; 3: 42. doi: 10.3389/fcrm.2016.00042

42. Ido A, Hasebe N, Matsuhashi H, et al. Coronary sinus occlusion enhances coronary collateral flow and reduces subendocardial ischemia. Am J Physiol Heart Circ Physiol. 2001; 280: H1361-H1367. doi: $10.3389 /$ fcvm.2016.00042

43. Konigstein M, Meyten N, Verheye S, et al. Transcatheter treatment for refractory angina with the coronary sinus reducer. EuroIntervention. 2014; 9(10): 1158-1164. doi: 10.4244/EIJV9I10A196

44. Banai S, Muvhar BS, Parikh KH, et al. Coronary sinus reducer stent for the treatment of chronic refractory angina pectoris: A prospective, open-label, multicenter, safety feasibility first-inman study. J Am Coll Cardiol. 2007; 49: 1783-1789. doi: 10.1016/j. jacc.2007.01.061

45. REDUCER-I: An Observational Study of the Neovasc Reducer ${ }^{\mathrm{TM}}$ System (2016). Web site. http://clinicaltrials.gov/ct2. (Identification No. NCT02710435).

46. Melzack R, Wall PD. Pain mechanisms: A new theory. Science. 1965; 150: 971-979. doi: 10.1126/science.150.3699.971

47. Eddicks S, Maier-Hauff K, Schenk M, et al. Thoracic spinal cord stimulation improves functional status and relieves symptoms in patients with refractory angina pectoris: The first placebo controlled randomized study. Heart. 2007; 93: 585-590.

48. Lanza G, Grimaldi R, Greco S, et al. Spinal cord stimulation for the treatment of refractory angina pectoris: A multicenter randomized single-blind study (the SCS-ITA trial). Pain. 2011; 152: 45-52. 
doi: 10.1016/j.pain.2010.08.044

49. Zipes D, Svorkdal N, Berman D, et al. C. Spinal cord stimulation therapy for patients with refractory angina who are not candidates for revascularization. Neuromodulation. 2012; 15: 550-558. doi: 10.1111/j.1525-1403.2012.00452.x

50. West PD, Colquhoun DM. TENS in refractory angina pectoris. Three case reports. Med J Aust. 1993; 158(7): 488-489.

51. Schmid JP, Capoferri M, Wahl A, et al. Cardiac shock wave therapy for chronic refractory angina pectoris. A prospective placebo-controlled randomized trial. Cardiovasc Ther. 2013; 31(3): e1-e6. doi: 10.1111/j.1755-5922.2012.00313.x

52. Przyklenk K, Bauer B, Ovize M, et al. Regional ischemic 'preconditioning' protects remote virgin myocardium from subsequent sustained coronary occlusion. Circulation. 1993; 87(3): 893-899. doi: 10.1161/01.CIR.87.3.893

53. Effect of Daily Remote Ischemic Conditioning on the Life Quality of Refractory Angina Pectoris Patients (D-RIC-RAP) (2015). Web site. http://clinicaltrials.gov/ct2. (Identification No. NCT02499250).

54. Moore RK, Groves D, Bateson S, et al. Health related quality of life of patients with refractory angina before and one year after enrolment onto a refractory angina program. Eur J Pain. 2005; 9: 305-310. doi: 10.1016/j.ejpain.2004.07.013

55. Fihn SD, Gardin JM, Abrams J, et al. 2012 ACCF/AHA/ACP/ AATS/PCNA/SCAI/STS guideline for the diagnosis and management of patients with stable ischemic heart disease. J Am Coll Cardiol. 2012; 60(24): e44-e164. doi: 10.1016/j.jacc.2012.07.013 